\title{
СИСТЕМАТИЗАЦИЯ ПРОЦЕССА УСВОЕНИЯ МАТЕРИАЛА ПО ТЕМЕ «ИНТЕГРАЛЬНОЕ ИСЧИСЛЕНИЕ» (МЕТОДИЧЕСКИЙ АСПЕКТ)
}

\author{
Виноградова Ю.А. ${ }^{1}$, Иванова О.К. ${ }^{1}$, Яновская Е.А. ${ }^{1}$ \\ ${ }^{1}$ ФБОУ ВО «Московский государственный технологический университет “СТАНКИН”», Москва, е-таіl: \\ oksivgor@mail.ru \\ В современных условиях основным результатом образовательной деятельности является не только \\ получение знаний, но и способность самостоятельно мыслить, способность к анализу и поиску решения \\ поставленной задачи. Математика развивает умение структурировать данные, создавать \\ математическую модель ситуации, анализировать и интерпретировать полученные результаты. Целью \\ обучения математике является формирование фундаментальных знаний, умений и навыков, \\ обеспечивающих прочное и сознательное овладение учащимися курсами математики и смежных \\ дисциплин в системе высшего образования. Интегральное исчисление - раздел математического \\ анализа, в котором изучаются интегралы, их свойства, способы вычисления и приложения. Этот раздел \\ наиболее успешно развивает у учашегося склонность к творческому, а также инженерному мышлению, \\ но наряду с этим вызывает большие трудности. Основной задачей преподавателей, работающих на \\ начальном этапе обучения в высшей школе, является предложение студентам всевозможных путей \\ эффективной адаптации к условиям образовательной среды вуза. Данная статья, основанная на \\ педагогическом опыте преподавания математики в техническом вузе, поможет систематизировать \\ некоторые темы раздела «Интегральное исчисление» доступно и наглядно и преодолеть указанные \\ трудности.
}

Ключевые слова: методика преподавания математики, интегральное исчисление, технический вуз, студент

\section{SYSTEMATIZATION OF THE PROCESS OF ASSOCIATION OF MATERIAL ON THE THEME «INTEGRAL CALCULUS» (METHODICAL ASPECT)}

\author{
Vinogradova Yu.A. ${ }^{1}$, Ivanova O.K. ${ }^{1}$, Yanovskaya E.A. ${ }^{1}$ \\ ${ }^{1} M S T U$ «STANKIN», Moscow,e-mail: oksivgor@mail.ru
}

In modern conditions, the main result of educational activity is not only the acquisition of knowledge, but also the ability to think independently, the ability to analyze and find solutions to the task. Mathematics develops the ability to structure data, create a mathematical model of the situation, analyze and interpret the results. The goal of teaching mathematics is the formation of fundamental knowledge, proficiency and skills that ensure the steady and conscious mastery of the mathematics courses and related disciplines in the system of higher education. Integral calculus is a branch of mathematical analysis in which integrals, their properties, methods of calculation and applications are studied. This section most successfully develops a student's propensity for creative, as well as engineering thinking, but at the same time causes great difficulties for the student. The main task of teachers working at the initial stage of higher education is to offer students all possible ways of effective adaptation to the conditions of the educational environment of the university. This article, based on the pedagogical experience of teaching mathematics in a technical university, will help accessibly and visually to systematize some topics of the section «Integral calculus» and overcome these difficulties.

Keywords: methodology of teaching mathematics, integral calculus, technical college, student

Преподаватели технических вузов нередко сталкиваются с проблемой поиска новых подходов в образовании. Современный студент - человек творческий, свободный, легко ориентирующийся в сети Интернет, в связи с этим не очень трудолюбивый. Преобладание традиционного подхода, при котором главным является передача знаний от преподавателя к учащемуся, зачастую приводит к формированию пассивной аудитории, не готовой слушать и воспринимать информацию. Тем более, говоря о студентах первого курса технического вуза, можно предположить, что трудности обучения связаны еще и с проблемой перехода с одной 
ступени образования на другую. Высшая математика в техническом вузе занимает вполне определенное положение. Студент любого технического вуза знает, что в основе всех его специальных технических предметов лежит серьезный курс высшей математики и что поэтому без знания высшей математики инженера из него не выйдет.

Целью нашего исследования является изучение педагогического опыта преподавания высшей математики и моделирование процесса математического образования студентов в период обучения на первом курсе технического вуза на примере Московского государственного университета «Станкин».

Сформулируем общие проблемы обучения [1]:

- несоответствие между увеличением объема учебной информации и фактором ограниченного времени обучения;

- $\quad$ интенсификация образовательного процесса;

- проблема формирования и активизации познавательных интересов учащегося;

- $\quad$ проблема целостного формирования личности.

Но в результате нашего исследования мы выявили также следующие проблемы:

• проблема доминанты алгоритмического мышления;

- ученический формализм в обучении;

- межпредметная раскоординированность учебного процесса;

- $\quad$ отсутствие достаточной мотивации к получению новых знаний;

- несоответствие дидактических целей высшего технического образования целям, сформулированным учащимся на основе здравого смысла (опыта учащегося).

Для решения указанных проблем, а также для облегчения процесса адаптации в вузе и для упрощения процесса обучения во многих вузах, в частности в МГТУ «Станкин», создана электронная образовательная среда, в которой размещены материалы по предметам в электронном виде: рабочие программы дисциплин, конспекты лекций, методические указания для обучающихся, электронное тестирование и многое другое, что позволяет упростить процесс самостоятельной работы студента. В то же время в процессе аудиторной работы преподаватель должен найти приемы, которые позволят улучшить восприятие той или иной информации. В вузе процесс обучения отличается более осознанным подходом к будущему знанию (прагматический мотив), но само обучение, кроме навыков, полученных в средней школе, требует развития качества самоанализа, самоидентификации изменяющейся среде. Подходя к процессу обучения как к системе, организующей мышление, память, внимание, мы в нем выделяем решение задач, сформулированных в любой форме [2].

Когнитивная деятельность в процессе решения задач в вузовской среде имеет весьма широкие границы: от сбора информации об оборудовании и проведения эксперимента до 
теоретико-философских обобщений результатов в абстрактных и технических науках. Следовательно, мотивированная активность связана с приобретением, организацией и использованием знания. Решение задач в математике обладает одним из самых главных преимуществ - возможностью моделировать процесс мышления, формализовать способ решения, иметь акт эксперимента в виде решения, представленного самим испытуемым. Рассматривая процесс поиска студентами решения определенной математической задачи, можно обнаружить, что сложность состоит не только в отсутствии знаний, но и в неумении их извлекать и применять [3, с. 206].

На примере темы «Интегральное исчисление» попробуем проанализировать подход к систематизации знаний. Интегрирование функций является отличной основой для развития творческой деятельности через самостоятельную работу.

По окончании изучения различных методов интегрирования нередко студент не в состоянии понять, какой метод в каком случае применяется. Для систематизации методов интегрирования студентам предлагается найти отличия в решении следующих примеров [4]:

1) $\quad \int \frac{d x}{x+1}$

2) $\quad \int \frac{d x}{x^{2}+1}$

3) $\quad \int \frac{x d x}{x+1}$

4) $\quad \int \frac{x d x}{x^{2}+1}$

5) $\quad \int \frac{x^{2} d x}{x^{2}+1}$

6) $\int \frac{x^{2} d x}{x+1}$

На первый взгляд при невнимательном рассмотрении студенты говорят, что эти примеры мало чем отличаются и выглядят не очень сложно. Но в процессе решения учащемуся приходится вспомнить не только таблицу интегралов (пример 2), но и применить методы, изученные ранее: свойства дифференциала (пример 1), подведение под знак дифференциала (пример 4), интегрирование неправильных рациональных дробей (примеры $3,5,6)$.

Здесь рассмотрим подробнее решение примера 3. Нередко можно заметить, что студенты указанную задачу решают разными способами: 
Способ 1. Выделение целой и дробной части

$$
\int \frac{x d x}{x+1}=\int \frac{(x+1-1) d x}{x+1}=\int\left(1-\frac{1}{x+1}\right) d x=x-\ln |x+1|+C
$$

Но иногда видим, что студент делает замену переменной:

Способ 2.

$$
\int \frac{x d x}{x+1}=\left|\begin{array}{l}
x+1=t \\
x=t-1 \\
d x=d t
\end{array}\right|=\int \frac{t-1}{t} d t=\int\left(1-\frac{1}{t}\right) d t=t-\ln |t|+C=x+1-\ln |x+1|+C .
$$

Второй способ хоть и громоздкий, все же требует задействования более сложных навыков: здесь требуется грамотно ввести замену и перейти обратно к исходной переменной.

На следующем занятии к этим примерам можно добавить следующие для установления отличий [5]:

7) $\quad \int \frac{d x}{\sqrt{x+1}}$;

8) $\int \frac{d x}{\sqrt{x^{2}+1}}$

9) $\int \frac{x d x}{\sqrt{x^{2}+1}}$

10) $\int \frac{d x}{x \sqrt{x^{2}+1}}$.

Если первые шесть интегралов могут особых проблем и не вызвать, а лишь помочь структурировать методы интегрирования, помочь прочувствовать различия применения подходов к решению, то интегралы с 7-го по 10-й уже имеют своей целью выбор оптимального способа решения, тем более у учащихся чаще всего присутствует страх перед иррациональностью. И если интегралы 7 и 8 еще достаточно просты (хотя имеет место проблема, когда студент сделал огромные преобразования, в процессе которых пришел к интегралам типа 7 и 8, и не может сообразить, что делать дальше), то интегралы 9 и 10 требуют задействования многих механизмов мышления.

Остановимся подробнее на примере 10. Как известно, существует несколько способов решения подобного примера, и здесь студенту предлагается выбор.

Способ 1. Подстановка $x=\operatorname{tg} t$, в таком случае, пользуясь тригонометрической формулой $1+\operatorname{tg}^{2} t=\frac{1}{\cos ^{2} t}$, получим, что $x^{2}=\operatorname{tg}^{2} t, 1+x^{2}=1+\operatorname{tg}^{2} t=\frac{1}{\cos ^{2} t}$, откуда 


$$
\begin{aligned}
& \sqrt{1+x^{2}}=\frac{1}{\cos t}, d x=\frac{d t}{\cos ^{2} t} \text {. В итоге имеем: } \\
& \int \frac{d x}{x \sqrt{x^{2}+1}}=\int \frac{\frac{d t}{\cos ^{2} t}}{\operatorname{tg} t \cdot \frac{1}{\cos t}}=\int \frac{d t}{\sin t}, \text { полученный интеграл можно решить с помощью }
\end{aligned}
$$

универсальной тригонометрической подстановки. Весь описанный способ очень трудоемкий, необходима двойная замена, вернуться к исходной переменной сложно, не зная соответствующих формул.

$$
\begin{aligned}
& \text { Способ 2. Подстановка } 1+x^{2}=t^{2} \text {. Тогда } x=\sqrt{t^{2}-1}, d x=\frac{t d t}{\sqrt{t^{2}-1}} \text {. В итоге имеем: } \\
& \int \frac{d x}{x \sqrt{x^{2}+1}}=\int \frac{\frac{t d t}{\sqrt{t^{2}-1}}}{\sqrt{t^{2}-1} \cdot t}=\int \frac{d t}{t^{2}-1} \text {. С помощью такой замены получили табличный }
\end{aligned}
$$
интеграл, решение получилось более коротким, но тем не менее нужно вернуться к исходной переменной, а это также может представлять трудность.

Способ 3. Вынесение из-под знака корня $x$ и внесение под знак дифференциала $\frac{1}{x^{2}}$. В итоге имеем:

$$
\int \frac{d x}{x \sqrt{x^{2}+1}}=\int \frac{d x}{x \cdot x \sqrt{1+\left(\frac{1}{x}\right)^{2}}}=-\int \frac{d\left(\frac{1}{x}\right)}{\sqrt{1+\left(\frac{1}{x}\right)^{2}}} . \text { Сделав замену } \frac{1}{x}=t, \text { придем к }
$$

интегралу 8. Проблемы могут возникнуть на этапе преобразований.

Если дать возможность прорешать этот пример всеми способами, рассмотреть поподробнее все возникающие в процессе решения проблемы, можно избежать сложностей в будущем.

Здесь еще рассмотрим пример $\int \frac{2 x-3}{x^{2}+4 x-5} d x$. Предложим решить его студенту разными способами [6].

Способ 1. Разложим знаменатель на множители:

$$
\begin{aligned}
& x^{2}+4 x-5=0 \\
& x_{1}=-5 ; x_{2}=1 \\
& x^{2}+4 x-5=(x+5)(x-1) .
\end{aligned}
$$


$\frac{2 x-3}{x^{2}+4 x-5}=\frac{2 x-3}{(x+5)(x-1)}=\frac{A_{1}}{x+5}+\frac{A_{2}}{x-1}=\frac{A_{1}(x-1)+A_{2}(x+5)}{(x+5)(x-1)}$.

Приравняем числители левой и правой дробей, получим $2 x-3=A_{1}(x-1)+A_{2}(x+5)$.

При $x=1-1=6 A_{2} \Rightarrow A_{2}=-\frac{1}{6}$.

При $x=-5-13=-6 A_{1} \Rightarrow A_{1}=\frac{13}{6}$.

То есть $\frac{2 x-3}{x^{2}+4 x-5}=\frac{\frac{13}{6}}{x+5}+\frac{-\frac{1}{6}}{x-1}$.

Вернемся к интегралу:

$$
\begin{aligned}
& \int \frac{2 x-3}{x^{2}+4 x-5} d x=\int\left(\frac{\frac{13}{6}}{x+5}+\frac{-\frac{1}{6}}{x-1}\right) d x=\frac{13}{6} \int \frac{d x}{x+5}-\frac{1}{6} \int \frac{d x}{x-1}= \\
& =\frac{13}{6} \ln |x+5|-\frac{1}{6} \ln |x-1|+C .
\end{aligned}
$$

Способ 2. Выделим в числителе производную знаменателя

$$
\begin{aligned}
& \int \frac{2 x-3}{x^{2}+4 x-5} d x=\int \frac{(2 x+4)-7}{x^{2}+4 x-5} d x=\int \frac{2 x+4}{x^{2}+4 x-5} d x-\int \frac{7}{x^{2}+4 x-5} d x= \\
& =\int \frac{d\left(x^{2}+4 x-5\right)}{x^{2}+4 x-5}-\int \frac{7}{\left(x^{2}+4 x+4\right)-9} d x=\ln \left|x^{2}+4 x-5\right|-\int \frac{7}{(x+2)^{2}-9} d(x+2)= \\
& =\ln \left|x^{2}+4 x-5\right|-\frac{7}{6} \ln \left|\frac{x+2-3}{x+2+3}\right|+C=\ln \left|x^{2}+4 x-5\right|-\frac{7}{6} \ln \left|\frac{x-1}{x+5}\right|+C .
\end{aligned}
$$

Заметим, что в процессе решения любым из способов мы приходим в процессе преобразований к интегралам типа 1-6.

Неизбежны трудности и при интегрировании тригонометрических выражений. Здесь тоже полезно использовать несколько способов решения одного и того же задания [7].

Например, рассмотрим интеграл $\int \operatorname{tg}^{2} x d x$.

Способ 1. Применение школьных тригонометрических формул:

$$
\int \operatorname{tg}^{2} x d x=\int \frac{\sin ^{2} x d x}{\cos ^{2} x}=\int \frac{\left(1-\cos ^{2} x\right) d x}{\cos ^{2} x}=\int \frac{d x}{\cos ^{2} x}-\int d x=\operatorname{tg} x-x+C .
$$


Способ 2. Подстановка $\operatorname{tg} x=t$, тогда $x=\operatorname{arctg} t, d x=\frac{d t}{1+t^{2}}$. В итоге имеем: $\int \operatorname{tg}^{2} x d x=\int \frac{t^{2} d t}{t^{2}+1}$, а это интеграл номер 5 .

Предлагая студенту на выбор один из способов, мы развиваем не только математическое мышление, но и творчество, необходимое для жизни в целом. Суть указанных примеров в том, чтобы студент видел перед глазами функциональную систему действий, смог указать последовательность их выполнения, тренировать умственную и речевую формулировки. Тем самым формируется организация последовательности действий и структуры результата. Исследовательские навыки, полученные в курсе математического анализа, оказывают положительное влияние на характер всей учебной деятельности обучающегося.

Таким образом, мы видим, что при установлении разницы между интегралами типа 16 студент может избежать трудностей при решении примеров типа 7-10, а также находить новые способы решения поставленных задач [8].

В результате использования описанной систематизации методов интегрирования существенно повышаются математическая грамотность студентов, качество формирования логического мышления. Практическая реализация указанных методов позволяет воспитать более качественного специалиста.

\section{Список литературы}

1. Ахмедзянова Ф.К. Формирование мотивации учения студентов ВУЗ в условиях концентрированного обучения. дис. ... канд. пед. наук. Казань, 2002. 167 с.

2. Тихомиров О.К. Психология мышления. М.: Издательство Московского университета, 1984. $272 \mathrm{c}$.

3. Лунгу К. Н. Систематизация приемов учебной деятельности студентов при обучении математике. М.: Книжный дом «Либроком», 2010. 424 с.

4. Сосенушкин Е.Н., Кадымов В.А., Иванова О.К., Яновская Е.А. Математическое обеспечение моделирования процессов пластического деформирования. Дифференциальное и интегральное исчисление: М.: Янус-К, 2018. 128 с.

5. Петросян Н.С., Холщевникова Н.Н., Шуманская Л. Б. Задачи и контрольные вопросы по математике для студентов 2 семестра. М.: МГТУ «СТАНКИН», 2008. 93 с.

6. Бубнова Т.В., Виноградова Ю.А., Господинова А.Г. Интегральное исчисление: учеб. пособие. М.: Янус-К, 2016. 90 с. 
7. Ахмедов Р.Э., Кадымов В.А. Функции одной независимой переменной: неопределенный интеграл и методы интегрирования, определенный интеграл и его приложения. Учебно-методическое пособие. Ч.2. М.: МГГЭУ, 2017. 62 с.

8. Иванов И.В. Олимпиады по математике для студентов вузов. Калуга: РГАУ-МСХА (КФ), 2010. $80 \mathrm{c.}$ 